\title{
ACEPTACIÓN DEL E-COMMERCE EN COLOMBIA: UN ESTUDIO PARA LA CIUDAD DE MEDELLÍN*
}

\author{
JUAN F. TAVERA MESÍAS**, JUAN C. SÁNCHEZ GIRALDO*** \& BERNARDO BALLESTEROS DÍAZ**** \\ UNIVERSIDAD DE ANTIOQUÍA
}

Recibido/ Received/ Recebido: 17-08-2011 - Aceptado/ Accepted / Aprovado: 02-11-2011

\begin{abstract}
Resumen
El e-Commerce es un fenómeno creciente en Latinoamérica y Colombia por lo que el estudio de su aceptación tecnológica es de alta importancia académica y empresarial. El objetivo de este artículo es identificar los antecedentes de la intención de uso de e-Commerce en Colombia. El Modelo de Aceptación Tecnológica (TAM), es complementado en este estudio con los constructos de Confianza y Seguridad Percibida para proponer un modelo ajustado al caso colombiano. Dicho modelo es contrastado empíricamente con una muestra de consumidores de la ciudad de Medellín. Se evidencia la importancia de la confianza y la utilidad percibida como antecedentes directos de la intención de uso del e-Commerce. Los resultados llenan vacíos existentes en la literatura sobre el e-Commerce en Colombia y permiten identificar implicaciones empresariales relevantes para el desarrollo de actividades comerciales a través de la Internet.
\end{abstract}

Palabras clave: E-Commerce, Comercio electrónico, Aceptación tecnológica, Tam, Comportamiento en línea.

\section{E-COMMERCE ACCEPTANCE IN COLOMBIA: A STUDY FOR MEDELLIN CITY}

\begin{abstract}
E-Commerce is an increasing phenomenon in Latin America and Colombia, that is why, its technological acceptance is very important academically and for business. The objective of this article is to identify the e-commerce use background in Colombia. Technological Acceptance Model (TAM) is complemented in this study with constructs of confidence and perceived security to propose an adjusted model for Colombian case. This model is empirically contrasted with a consumers sample
\end{abstract}

* Resultado de investigación realizada por el iMARK-Grupo de Investigación en Marketing y Docente del Departamento de Administración de Empresas de la Universidad de Antioquia (Medellín, Colombia).

** Candidato a Doctor en Marketing y Máster Oficial en Marketing e Investigación de Mercados de la Universidad de Valencia. Magíster en Administración de la Universidad EAFIT. Actualmente es Coordinador de iMARK-Grupo de Investigación en Marketing y Docente del Departamento de Administración de Empresas de la Universidad de Antioquia. Correo electrónico: jftavera@economicas.udea. edu.co

**** Estudiante de Maestría en Mercadeo de la Universidad de Manizales y Especialista en Negocios Internacionales de la Fundación Universitaria ESUMER. Actualmente es miembro de iMARK-Grupo de Investigación en Marketing y Docente del Departamento de Administración de Empresas de la Universidad de Antioquia. Correo electrónico: jcsanchezg@economicas.udea.edu.co

****** Magíster en Administración de la Universidad EAFIT. Especialista en Gerencia de la Universidad Pontificia Bolivariana. Actualmente es miembro de iMARK-Grupo de Investigación en Marketing y Docente del Departamento de Administración de Empresas de la Universidad de Antioquia. Correo electrónico: bballesteros@economicas.udea.edu.co 
from Medellín. It is evident the importance of confidence and perceived benefit as direct background of the intention of using e-Commerce, and the ease of use and perceived security as indirect background. Results fill gaps in literature on e-commerce in Colombia and allow identifying relevant business implications for commercial activities development through the internet.

Keywords: e-Commerce, Technology acceptance, Technology acceptance model, TAM, On-line behavior.

\title{
ACEITAÇÃO DO E-COMMERCE NA COLÔMBIA: UM ESTUDO PARA A CIDADE DE MEDELLÍN
}

\begin{abstract}
Resumo
O e-Commerce é um fenômeno crescente na América Latina e na Colômbia. Por isto o estudo da sua aceitação tecnológica é de alta importância acadêmica e empresarial. O objetivo deste artigo é identificar os antecedentes da intenção de uso de e-Commerce na Colômbia. O Modelo de Aceitação Tecnológica (TAM) é complementado neste estudo pelos constructos de Confiança e Segurança Percebida com o fim de propor um modelo adequado ao caso colombiano. Referido modelo é contrastado empiricamente com uma mostra de consumidores da cidade de Medellín. Com isto se evidencia a importância da confiança e da utilidade percebida como antecedentes diretos da intenção do uso do e-Commerce, e a facilidade do uso e da segurança percebida como antecedentes indiretos. Os resultados preenchem vazios existentes na literatura sobre o e-Commerce na Colômbia e permitem identificar envolvimentos empresariais relevantes para o desenvolvimento de atividades comerciais através da Internet.
\end{abstract}

Palauras chave: e-Commerce, Comércio eletrônico, Aceitação tecnológica, TAM, Comportamento em linha.

Tavera, J.; Sancjez, J. \& Ballesteros, B. (2011) Aceptación del e-commerce en Colombia: un estudio para la ciudad de Medellín. En: Revista de la Facultad de Ciencias Económicas de la Universidad Militar Nueva Granada. rev.fac.cienc.econ, XIX (2)

JEL: L81, L99, M13.

\section{Introducción}

En Colombia el Internet ha penetrado significativamente el mercado, identificándose para el primer trimestre del año 2011 un total de 5.054 .877 suscripciones de Internet, de las cuales el $61 \%$ corresponden a Internet fijo y el $39 \%$ restante a Internet móvil (Ministerio de Tecnologías de la Información y las Comunicaciones, 2011). El estudio de Microsoft Advertising revela adicionalmente, que la cifra de usuarios de Internet es muy superior a la de suscripciones, identificando más de 25 millones de usuarios, para una penetración total del 54\%, la segunda mayor de Latinoamérica (Mejía, 2011).

La Internet es una de esas tecnologías que ha desencadenado transformaciones en los hábitos y costum- bres de los individuos (Castells, 2000). Esta ofrece, entre otras cosas, la infraestructura necesaria para el intercambio de información y la realización de transacciones comerciales (Al-Gahtani, 2011). La Internet y sus desarrollos tecnológicos han permitido el desarrollo de una nueva economía digital que facilita nuevos espacios para el desarrollo del objeto social de las empresas y la satisfacción de los deseos y necesidades de los clientes. El e-Commerce o comercio electrónico, comprendido como el desarrollo de procesos de compra y venta soportados por medios electrónicos y especialmente por la Internet (Kotler \& Armstrong, 2003), se ha encargado de soportar el crecimiento de la nueva economía.

Grewal \& Levy (2009) identifican el crecimiento del Internet y el e-Commerce como una de las cuatro 
áreas con mayor potencial de contribución a la investigación sobre el retail, sin embargo la investigación en países en desarrollo se encuentra en una fase temprana de desarrollo teórico (Kshetri, 2007). La literatura sobre el tema del comercio electrónico en Colombia es bastante escasa y específicamente nula en el caso de la aceptación tecnológica. Por lo anterior, es de gran importancia indagar acerca de los antecedentes por los que los individuos se deciden a adoptar este tipo de tecnología para llegar a comprenderlos y darles aplicación científica y práctica para el desarrollo de nuevos negocios o mejoramiento de los actuales.

El presente artículo hace uso del Modelo de Aceptación Tecnológica - TAM (Davis, 1989; Davis et al., 1989) para abordar el fenómeno de la aceptación tecnológica del e-Commerce en Colombia. Dicho modelo ha sido de amplio uso en numerosos mercados y tecnologías (Ji-Won \& Young-Gul, 2001; Khalifa \& Shen, 2008; Lu' et al., 2003; Luarn \& Lin, 2005; Robinson et al., 2005; Savitskie et al., 2007; Venkatesh \& Davis, 2000; Wang et al., 2006; Wu \& Wang, 2005; Yang, 2005, 2007; Yu et al., 2005) y tal como propone Al-Gahtani (Al-Gahtani, 2011), se puede hacer uso de otras experiencias adaptándolas a modelos propios, por lo que se utilizará dicho modelo para el caso colombiano.

El e-Commerce propone ventajas y desventajas frente al comercio tradicional. En primera instancia facilita el acceso a información para llevar a cabo una transacción, reduce costos de búsqueda de información entre los compradores y los vendedores, genera mayor transparencia en los costos, acorta la distancia espacial entre compradores y vendedores, reduce el tiempo de compra y posesión de los productos (Varadarajan \& Yadav, 2002). Sin embargo, la Internet genera mayor incertidumbre en las transacciones en línea (Al-Gahtani, 2011; Brynjolfsson \& Smith, 2000; Kim et al., 2008) y esto hace que la confianza sea importante para el comercio electrónico (Doney et al., 2007; Liu et al., 2005). La confianza es decisiva y más aún cuando hay una separación espacial y temporal entre compradores, vendedores $y$ productos en la infraestructura de internet (Brynjolfsson \& Smith, 2000). Lo anterior conlleva a que el modelo propuesto incluya la confianza como un elemento antecedente del comportamiento de aceptación tecnológica que complementa al TAM, y a su vez se propone la seguridad percibida como antecedente de la confianza.

Tras lo anterior, en el presente estudio se busca responder a la pregunta de investigación ¿Qué constructos determinan la aceptación de e-Commerce en Colombia? Para esto se planteó como objetivo identificar los principales antecedentes de la intención de uso del e-Commerce a través de los modelos y variables planteadas y se desarrolló el modelo mencionado contrastándolo con una muestra empírica, en la ciudad de Medellín. El análisis factorial confirmatorio permitió asegurar la fiabilidad y validez de las escalas de medida y la modelación por ecuaciones estructurales, obtuvo un buen ajuste y el no rechazo de muchas de las hipótesis planteadas.

\subsection{Modelo de Aceptación Tecnológica}

El Modelo de Aceptación Tecnológica (TAM-Technology Acceptance Model) planteado por Davis (1989) pretende dar explicación al comportamiento humano, relacionado con la intención comportamental, como principal determinante del uso de tecnologías (Bigné et al., 2007; Davis, 1989; Davis et al., 1989; Lu' et al., 2003; Taylor \& Todd, 1995; Venkatesh \& Davis, 2000; Venkatesh et al., 2003; Wu \& Wang, 2005). Según Rodríguez del Bosque \& Herrero (2007), el TAM constituye una adaptación de la Teoría de Acción Razonada (TRA - Theory of Reasoned Action) -(Ajzen \& Fishbein, 1977; Fishbein \& Ajzen, 1975) - centrada en la conducta de uso de nuevas tecnologías. En particular, esta teoría presta especial atención al análisis del efecto de factores externos sobre las creencias, actitudes e intenciones de los individuos (Davis et al., 1989). De este modo, el Modelo de Aceptación de Tecnología identifica dos creencias concretas que afectan de forma fundamental a la aceptación de innovaciones informáticas: la utilidad percibida y la facilidad de uso percibida (Davis, 1989; Davis et al., 1989; Rodríguez del Bosque \& Herrero, 2007)

En el TAM, la facilidad percibida de uso y la utilidad percibida son las principales variables determinantes del uso de la tecnología, ejerciendo su influencia a 
través de la actitud hacia el uso, determinante de la Intención (Davis, 1989; Davis et al., 1989; King \& $\mathrm{He}, 2006)$.

Los conceptos de Intención de uso y actitud provienen de la Teoría de Acción Razonada (Fishbein \& Ajzen, 1975), en la cual se explican los comportamientos a partir de la existencia de la intención de ejecutar los mismos. Dicha intención de comportarse plantea dos antecedentes: la actitud hacia la ejecución del comportamiento y la norma subjetiva. El TAM recoje estos conceptos y entiende la intención como un constructo actitudinal previo al comportamiento que indica el propósito de realizar el mismo. Por su parte, la actitud se entiende como la predisposición para responder consistentemente de una manera favorable o desfavorable a un objeto (Ajzen \& Fishbein, 1977; Davis et al., 1989; Fishbein \& Ajzen, 1975).

Por su parte, la utilidad percibida es entendida como la probabilidad subjetiva del prospecto usuario de que el uso de la tecnología en cuestión mejorará su desempeño (Davis, 1989; Davis et al., 1989) y la Facilidad de uso percibida como el grado en el que el prospecto usuario espera que el uso de dicha tecnología esté desprovisto de esfuerzo (Davis, 1989; Davis et al., 1989; Venkatesh, 2000; Venkatesh \& Davis, 1996). A su vez, la utilidad percibida es antecedente de la actitud y de la intención de uso, mientras que la Facilidad de uso percibida es antecedente de la actitud y de la utilidad percibida.

La Ilustración 1 representa gráficamente la relación entre las variables del modelo TAM tal como se señaló anteriormente.
Dicho modelo ha sido aplicado con buenos resultados a la aceptación de sistemas de información (Davis, 1989; Davis et al., 1989; Mathieson et al., 2001), al comercio móvil (Khalifa \& Shen, 2008; Simon et al., 2006; Wang et al., 2006; Wu \& Wang, 2005), la banca electrónica (Lassar et al., 2005; Luarn \& Lin, 2005; Xiaoni et al., 2006) y también al comercio electrónico en diversos mercados a nivel mundial (Garrity et al., 2007; Jones \& Leonard, 2007; Kleijnen et al., 2004; Pavlou, 2001; Savitskie et al., 2007; Shih, 2004; Singh et al., 2006).

De acuerdo con lo anterior se proponen las siguientes hipótesis, que corresponden al modelo básico propuesto en el TAM, así:

H1: Facilidad percibida de uso influye sobre la Utilidad Percibida

H2: Facilidad percibida de uso influye sobre la Actitud hacia la realización de compras/transacciones por internet

H3: Utilidad percibida influye sobre la actitud hacia la realización de compras/transacciones por internet

H4: Utilidad percibida influye sobre la Intención de uso del internet para la realización de compras/transacciones

H5: Actitud hacia la realización de compras/ transacciones por internet influye sobre la Intención de uso de internet para la realización de compras/transacciones.

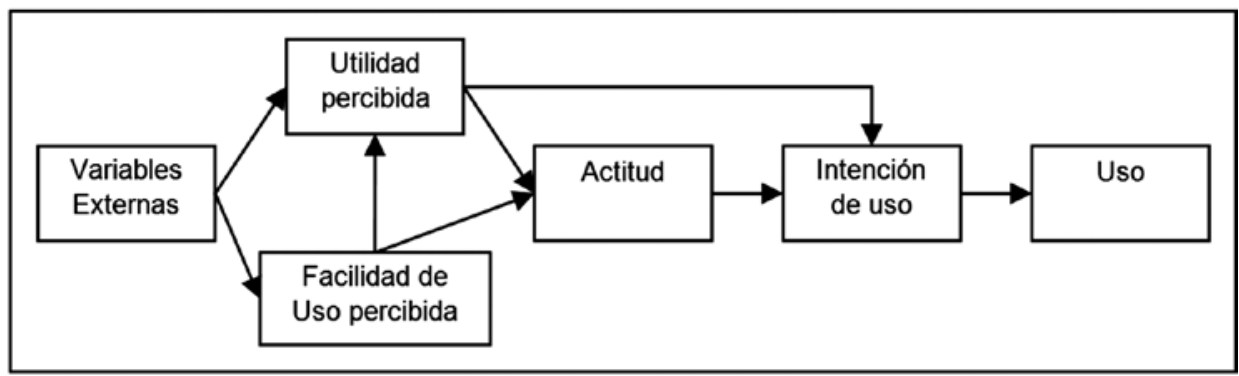

Ilustración 1. Modelo de Aceptación Tecnológica (TAM) ${ }^{1}$

1 Fuente: Davis et al. (1989). 


\subsection{La Confianza y la Seguridad percibidas en el comercio electrónico}

La confianza juega un papel crucial para el marketing relacional, es decir, para la construcción de relaciones duraderas en el largo plazo, especialmente en situaciones donde una de las partes perciben riesgo, incertidumbre o interdependencia (Palvia, 2009). La ausencia de control sobre las acciones de otro o conocer completamente sus motivaciones conduce a que los individuos inhiban sus intenciones de desarrollar comportamientos (Gefen, 2000).

Desde finales de los años cincuenta, Deutsch (1958) estudiaba el fenómeno de la confianza en la negociación, entendiendo la misma como un conjunto de expectativas que influyen sobre la intención de comportamiento del individuo dado que involucra la posibilidad de tener pérdidas en la relación con la otra parte (Deutsch, 1958). Moorman et al. (1992) define el concepto para el marketing relacional como el deseo de fiarse de la otra parte presente en un intercambio, de la cual se cree que cumplirá (Moorman et al., 1993). Por lo tanto, la confianza puede entenderse como la certeza que percibe un individuo de que las expectativas que posee sobre el comportamiento de la otra parte serán satisfechas (Gefen, 2000), cumpliendo así con sus promesas (Gefen et al., 2003). En el comercio, la confianza puede entenderse tanto como un conjunto de creencias sobre la otra parte del intercambio, así como una intención de comportamiento (Gefen, 2000; Gefen \& Straub, 2004; Moorman et al., 1993; Moorman et al., 1992), siendo ambas posibles antecedentes de la intención de efectuar una transacción (Liu et al., 2005; Palvia, 2009; Pavlou, 2001).

Adicionalmente, Fishbein \& Ajzen (1975) evidencian que las creencias acerca de la ejecución del comportamiento conllevan a la elaboración de evaluaciones positivas o negativas acerca del mismo, las cuales de forma agregada determinan la formación de una actitud hacia dicho comportamiento. En otras palabras, las creencias son antecedentes de las actitudes (Ajzen, 1991; Ajzen \& Fishbein, 1977; Ajzen \& Gilbert, 2008; Bagozzi, 1992; Fishbein \& Ajzen, 1975).

Lo anterior permite suponer que si la confianza puede entenderse como un conjunto de creencias, en- tonces se espera que éstas influyan, en el caso del e-Commerce, sobre la actitud de desarrollar transacciones a través de la Internet (Palvia, 2009).

Como se explicaba anteriormente, los consumidores se ven en situaciones complejas al enfrentarse al eCommerce, dadas las vulnerabilidades de la situación (Gefen, 2000; Gefen et al., 2003; Kim et al., 2011; Suh \& Han, 2003). La seguridad o control que perciban los individuos frente a una transacción mediante la Internet, será determinante para la generación de la confianza (Kim et al., 2011; Suh \& Han, 2003).

El estudio de Kim et al. (2011) demostró que la seguridad percibida es un importante antecedente de la confianza. Suh \& Han (2003), por su parte, evidenciaron que el hecho de que en las transacciones de e-Commerce las partes no puedan negar que han participado, influye sobre la confianza percibida. De igual forma, el que la información suministrada por los clientes no pueda ser interceptada o descifrada por terceros y que a su vez sea protegida y no entregada o publicada sin autorización, influye sobre la confianza percibida en el comercio electrónico (Suh \& Han, 2003) siendo estos componentes propios del constructo seguridad.

Por lo anterior se proponen las siguientes hipótesis adicionales al modelo TAM:

H6: La Seguridad Percibida influye sobre la Confianza

H7: La Confianza influye sobre la Actitud hacia la realización de compras/transacciones por internet

H8: La Confianza influye sobre la Intención de uso del internet para la realización de compras/ transacciones.

\subsection{Modelo propuesto}

Para el análisis y estudio se propone el modelo de la Ilustración 2, que recoge las hipótesis planteadas en la presente investigación, para proceder a validar el modelo y las relaciones estructurales planteadas mediante el estudio empírico. 


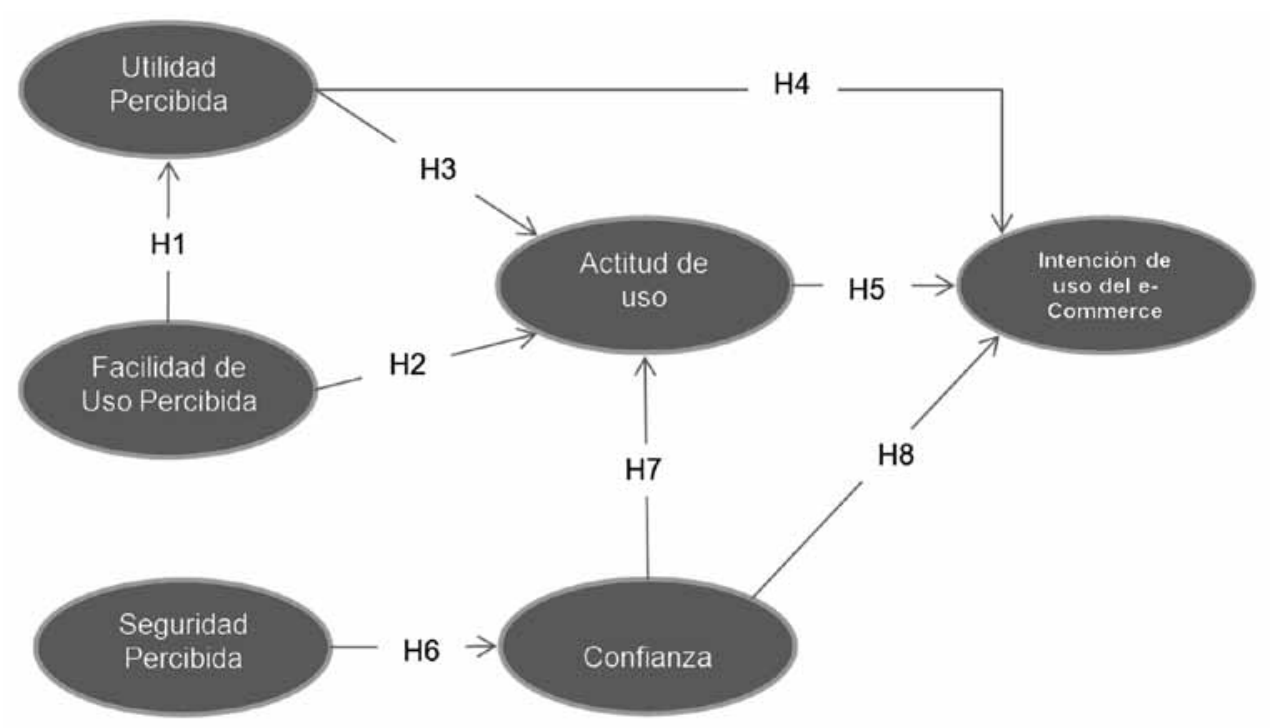

Ilustración 2. Modelo de Aceptación tecnológica del comercio electrónico²

Se ha utilizado el TAM como un modelo de referencia para evaluar los antecedentes de la intención de uso del e-commerce que tienen los consumidores Colombianos y se han adicionado los constructos Seguridad y Confianza percibida para complementar el TAM.

\section{Metodología}

La investigación se realizó en dos fases, exploratoria y descriptiva. La primera con el objetivo de definir adecuadamente el problema de investigación propuesto, elaborar el marco teórico y estado del arte necesario para la comprensión de dicho problema y el planteamiento de las hipótesis de investigación.
La segunda, de corte transversal simple, con el objetivo de dar explicación a dicho fenómeno haciendo uso de instrumentos de medición estructurados que fueron aplicados a una muestra de 497 individuos durante el mes de noviembre de 2010. La selección de la muestra fue por conveniencia, como numerosos estudios publicados sobre el mismo fenómeno en otros mercados (Gefen et al., 2003; Gefen y Straub, 2004; Jones \& Leonard, 2007; Kim et al., 2008; Liu et al., 2005; Palvia, 2009) y siguiendo el cumplimiento de cuotas (Kim et al., 2011) buscando que la muestra se ajustara a la distribución poblacional por edades y género en Colombia. La Tabla 1 recoge la ficha técnica del estudio.

Tabla 1. Ficha técnica del estudio desarrollado. ${ }^{3}$

\begin{tabular}{|l|l|}
\hline Unidad muestral & Usuarios de internet mayores de 14 años \\
\hline Ámbito de estudio & Medellín y el área Metropolitana \\
\hline Método de recogida de información & Entrevista personal \\
\hline Procedimiento de muestreo & Muestreo por conveniencia, siguiendo cuotas \\
\hline Tamaño muestral & 497 \\
\hline Fecha del trabajo de campo & Noviembre de 2010 \\
\hline
\end{tabular}

Fuente: Elaboración propia.

Fuente: Elaboración propia. 
La Tabla 2 resume las principales características de la muestra, destacándose la población con edad inferior a 35 años $(71,03 \%)$, una proporción algo mayor de género femenino $(54,73 \%)$ y de individuos solteros $(54,73 \%)$. Predominan los individuos con formación técnica, tecnológica o profesional (72,83\%), empleados $(56,94 \%)$ y con un nivel de ingresos entre un salario mínimo y COP $\$ 1.500 .000$ (52,42\%). Estudios anteriores indican que las muestras compuestas mayoritariamente por jóvenes y con buen nivel educativo favorecen los estudios relacionados con el comportamiento en línea (Bigné et al., 2007; Kim et al., 2008; Netsize, 2008).
Para la medición de los diferentes constructos del modelo, se empleó una escala de Likert de cinco posiciones en donde 1 indica "muy en desacuerdo", 2 "desacuerdo", 3 "neutral", 4 de acuerdo y 5 "muy de acuerdo".

La Tabla 3 resume cómo se ha realizado la medición de las variables en el estudio empírico, así como la procedencia de los ítems utilizados en las escalas de medida. Las variables utilizadas fueron traducidas y adaptadas para garantizar el entendimiento de las mismas por parte de los individuos entrevistados.

Tabla 2. Características de la muestra ${ }^{4}$

\begin{tabular}{|l|c|}
\hline \multicolumn{1}{|c|}{ VARIABLE } & $\begin{array}{c}\text { Medellín y el Área } \\
\text { Metropolitana } \\
\text { (Colombia) } \mathbf{n = 4 9 7}\end{array}$ \\
\hline Edad & \\
\hline De 14 a 24 años & $29,38 \%$ \\
\hline De 25 a 34 años & $41,65 \%$ \\
\hline De 35 a 49 años & $23,94 \%$ \\
\hline De 50 a 64 & $4,43 \%$ \\
\hline 65 0 mayor & $0,60 \%$ \\
\hline Género & \\
\hline Masculino & $45,27 \%$ \\
\hline Femenino & $54,73 \%$ \\
\hline Estado civil & \\
\hline Soltero & $2,013 \%$ \\
\hline Casada & $13,48 \%$ \\
\hline Con pareja & $5,03 \%$ \\
\hline Separada & $3,62 \%$ \\
\hline Divorciada & \\
\hline Viuda & \\
\hline Nivel de estudios & \\
\hline Menos de primaria / No lee & \\
\hline Bachillerato incompleto & \\
\hline Bachillerato completo & \\
\hline
\end{tabular}

\begin{tabular}{|l|c|}
\hline \multicolumn{1}{|c|}{ VARIABLE } & $\begin{array}{c}\text { Medellín y el Área } \\
\text { Metropolitana } \\
\text { (Colombia) } \mathbf{n = 4 9 7}\end{array}$ \\
\hline Técnica/Tecnología & $35,81 \%$ \\
\hline Profesional & $37,02 \%$ \\
\hline Especialista & $8,05 \%$ \\
\hline Maestría 0 Doctorado & $2,21 \%$ \\
\hline Ocupación & \\
\hline Estudiante & $26,76 \%$ \\
\hline Ama de casa & $1,81 \%$ \\
\hline Independiente & $9,86 \%$ \\
\hline Trabajador empleado & $56,94 \%$ \\
\hline Desempleado & $3,62 \%$ \\
\hline Jubilado & $1,01 \%$ \\
\hline Nivel de ingresos & \\
\hline No percibo un salario & $3,5,53 \%$ \\
\hline Menos de un salario mínimo & $15,69 \%$ \\
\hline Entre un salario mínimo y \$1.000.000 & $6,44 \%$ \\
\hline Entre $\$ 1.000 .001$ y $\$ 1.500 .000$ & $3,64 \%$ \\
\hline Entre $\$ 1.500 .001$ y $\$ 2.500 .000$ & \\
\hline Entre \$2.500.001 y $\$ 3.500 .000$ & \\
\hline Más de \$3.500.000 & \\
\hline
\end{tabular}

4 Fuente: Elaboración propia. 
Tabla 3. Escalas de medida utilizadas. 5

\begin{tabular}{|c|c|c|c|}
\hline CONSTRUCTO & CÓDIGO & ÍTEM & FUENTES \\
\hline \multirow{6}{*}{$\begin{array}{l}\text { Facilidad } \\
\text { de Uso } \\
\text { Percibida }\end{array}$} & FU1 & Aprender a utilizar el internet para realizar compras/transacciones es fácil para mí & \multirow{12}{*}{$\begin{array}{c}\text { Davis (1989); } \\
\text { Moore \& } \\
\text { Benbasat (1991) }\end{array}$} \\
\hline & FU2 & En conjunto, creo que realizar compras/transacciones por internet es fácil & \\
\hline & FU3 & Me resultaría fácil adquirir habilidad para realizar compras/transacciones por internet & \\
\hline & FU4 & No me supondría ningún esfuerzo mental realizar compras/transacciones por internet & \\
\hline & FU5 & Es fácil realizar compras/transacciones por internet como yo quiero & \\
\hline & FU6 & Mi interacción con internet cuando realizo compras/transacciones por internet es clara y comprensible & \\
\hline \multirow{6}{*}{$\begin{array}{l}\text { Utilidad } \\
\text { Percibida }\end{array}$} & UP1 & $\begin{array}{l}\text { Utilizar internet me permite realizar compras/transacciones más rápidamente que cuando lo hago en una } \\
\text { tienda }\end{array}$ & \\
\hline & UP2 & Utilizar internet para realizar compras/transacciones me permite ahorrar tiempo & \\
\hline & UP3 & Utilizar internet para realizar compras/transacciones ofrece beneficios & \\
\hline & UP4 & En conjunto, utilizar internet para realizar compras/transacciones es muy útil & \\
\hline & UP5 & Utilizar internet me permite realizar compras/transacciones de forma más eficiente que personalmente & \\
\hline & UP6 & Utilizar internet para realizar compras/transacciones me resulta más sencillo que personalmente & \\
\hline \multirow{4}{*}{$\begin{array}{c}\text { Actitud } \\
\text { hacia el } \\
\text { e-Commerce }\end{array}$} & A1 & Realiza compras/transacciones por internet es una idea que me gusta & \multirow{4}{*}{$\begin{array}{l}\text { Taylor \& Todd } \\
\quad(1995)\end{array}$} \\
\hline & A2 & Realiza compras/transacciones por internet me parece una idea inteligente & \\
\hline & A3 & Realiza compras/transacciones por internet es una buena idea & \\
\hline & A4 & Realiza compras/transacciones por internet es una idea positiva & \\
\hline \multirow{6}{*}{$\begin{array}{l}\text { Seguridad } \\
\text { Percibida }\end{array}$} & SP1 & $\begin{array}{l}\text { Me siento preocupado acerca de la privacidad de mi información personal durante una transacción por } \\
\text { internet }\end{array}$ & \multirow{6}{*}{$\begin{array}{l}\text { Chang \& Chen } \\
\text { (2009); Kim et } \\
\text { al. (2008); Kim } \\
\text { et al. (2011) }\end{array}$} \\
\hline & SP2 & Me siento seguro realizando transacciones en sitios web & \\
\hline & SP3 & $\begin{array}{l}\text { En general, proveer información de la tarjeta de crédito en sitios web es más riesgoso que en una compra } \\
\text { personal }\end{array}$ & \\
\hline & $\mathrm{SP} 4$ & Comprar en sitios web representa mayor riesgo financiero que una compra tradicional & \\
\hline & SP5 & Realizar pagos por internet es libre de riesgos & \\
\hline & SP6 & Mi privacidad sería garantizada en sitios web & \\
\hline \multirow{4}{*}{$\begin{array}{l}\text { Confianza } \\
\text { Percibida }\end{array}$} & $\mathrm{C} 01$ & Los sitios web donde se puede realizar compras/transacciones son confiables & \multirow{4}{*}{$\begin{array}{l}\text { Kim et al. } \\
\text { (2008); Kim et } \\
\text { al. (2011) }\end{array}$} \\
\hline & $\mathrm{CO2}$ & Los sitios web son confiables para proteger mi información personal & \\
\hline & $\mathrm{CO3}$ & $\begin{array}{l}\text { Los sitios web donde se puede realizar compras/transacciones dan la impresión que cumplen promesas } \\
\text { y compromisos. }\end{array}$ & \\
\hline & $\mathrm{CO4}$ & Los sitios web donde se puede realizar compras/transacciones tienen integridad & \\
\hline \multirow{5}{*}{$\begin{array}{c}\text { Intención } \\
\text { de usar el } \\
\text { e-Commerce }\end{array}$} & IU1 & Tengo la intención de realizar compras/transacciones por internet & \multirow{5}{*}{$\begin{array}{c}\text { Taylor \& Todd } \\
\text { (1995); Coyle \& } \\
\text { Thorson (2001); } \\
\text { Kim \& Biocca } \\
\text { (1997) }\end{array}$} \\
\hline & IU2 & Pienso que realizaré compras/transacciones por internet en los próximos meses & \\
\hline & IU3 & Recomendaré a otros que realicen compras/transacciones por internet & \\
\hline & IU4 & Usaría mis tarjetas de crédito/débito para realizar compras/transacciones en internet & \\
\hline & IU5 & Usaré frecuentemente sitios web para realizar compras/transacciones & \\
\hline
\end{tabular}

5 Fuente: Elaboración propia. 


\section{Resultados y discusión}

\subsection{Validación de escalas de medida}

La comprobación de la validez de las escalas de medida utilizadas fue desarrollada mediante un análisis factorial confirmatorio por medio del software EQS versión 6.1. El test de Mardia arrojó un coeficiente de 258,012 con un estimado normalizado de 70,167 indicando la presencia de kurtosis multivariante y la ausencia de normalidad en los datos. Por lo anterior, el método de estimación seleccionado fue el de máxima verosimilitud robusto.
Para lograr la convergencia del modelo fue necesario recurrir a la eliminación de los indicadores SP1, SP3 y SP4, dado que sus cargas factoriales estandarizadas eran inferiores a 0,6 siguiendo las recomendaciones de Bagozzi \& Yi (1988). Una vez depurado el modelo de medida todas las cargas factoriales estandarizadas fueron significativas y superaron el nivel de 0,6 (Bagozzi \& Yi, 1988), el promedio de las cargas de los indicadores sobre cada factor fue superior a 0,7 para todos los constructos (Hair et al., 2001) y las correlaciones de los indicadores con cada factor fue superior a 0,3 en todos los casos (Uriel \& Aldás, 2005), indicando la presencia de validez convergente, tal como se refleja en la Tabla 4, la correlación ítem-total fue alta para todos los casos, superior a 0,5.

Tabla 4. Análisis confirmatorio de la validez convergente ${ }^{6}$

\begin{tabular}{|c|c|c|c|c|c|c|}
\hline Constructo & Item & $\begin{array}{c}\text { Correlación } \\
\text { ítem-total }\end{array}$ & $\begin{array}{c}\text { Cargas } \\
\text { Factoriales } \\
\text { Estandarizadas }\end{array}$ & $\begin{array}{c}\text { Promedio Cargas } \\
\text { Factoriales } \\
\text { Estandarizadas }\end{array}$ & Valor $\mathbf{t}$ & $\begin{array}{l}\text { Correlación } \\
\text { de Pearson }\end{array}$ \\
\hline \multirow{6}{*}{ Facilidad de Uso (FU) } & FU1 & 0,752 & 0,792 & \multirow{6}{*}{0,819} & 21,849 & 0,627 \\
\hline & FU2 & 0,828 & 0,862 & & 26,491 & 0,744 \\
\hline & FU3 & 0,789 & 0,820 & & 20,240 & 0,672 \\
\hline & FU4 & 0,734 & 0,764 & & 21,034 & 0,584 \\
\hline & FU5 & 0,809 & 0,850 & & 25,704 & 0,723 \\
\hline & FU6 & 0,777 & 0,826 & & 25,551 & 0,683 \\
\hline \multirow{6}{*}{ Utilidad Percibida (UP) } & UP1 & 0,732 & 0,766 & \multirow{6}{*}{0,792} & 22,945 & 0,586 \\
\hline & UP2 & 0,745 & 0,786 & & 20,109 & 0,618 \\
\hline & UP3 & 0,764 & 0,830 & & 23,756 & 0,689 \\
\hline & UP4 & 0,806 & 0,875 & & 25,292 & 0,766 \\
\hline & UP5 & 0,750 & 0,770 & & 22,804 & 0,593 \\
\hline & UP6 & 0,704 & 0,725 & & 20,581 & 0,526 \\
\hline \multirow{4}{*}{ Actitud (A) } & $\mathrm{A} 1$ & 0,788 & 0,819 & \multirow{4}{*}{0,898} & 26,909 & 0,671 \\
\hline & $\mathrm{A} 2$ & 0,875 & 0,912 & & 27,430 & 0,833 \\
\hline & A3 & 0,895 & 0,939 & & 29,149 & 0,882 \\
\hline & A4 & 0,882 & 0,920 & & 25,770 & 0,847 \\
\hline \multirow{3}{*}{ Seguridad Percibida (SP) } & SP2 & 0,530 & 0,709 & \multirow{3}{*}{0,701} & 17,925 & 0,503 \\
\hline & SP5 & 0,562 & 0,626 & & 14,228 & 0,392 \\
\hline & SP6 & 0,625 & 0,769 & & 22,467 & 0,592 \\
\hline \multirow{4}{*}{ Confianza (CFZ) } & CFZ1 & 0,750 & 0,853 & \multirow{4}{*}{0,793} & 25,160 & 0,728 \\
\hline & CFZ2 & 0,754 & 0,854 & & 27,613 & 0,730 \\
\hline & CFZ3 & 0,689 & 0,718 & & 17,776 & 0,516 \\
\hline & CFZ4 & 0,715 & 0,745 & & 17,708 & 0,555 \\
\hline \multirow{5}{*}{ Intención de Uso (IU) } & IU1 & 0,821 & 0,851 & \multirow{5}{*}{0,869} & 32,119 & 0,724 \\
\hline & IU2 & 0,852 & 0,877 & & 33,950 & 0,769 \\
\hline & IU3 & 0,813 & 0,842 & & 28,284 & 0,708 \\
\hline & IU4 & 0,832 & 0,877 & & 32,428 & 0,769 \\
\hline & IU5 & 0,860 & 0,899 & & 34,827 & 0,808 \\
\hline
\end{tabular}

\footnotetext{
6 Fuente: Elaboración propia.
} 
El análisis de la validez discriminante se ejecutó mediante: (a) la comprobación de que el intervalo de confianza en la estimación de la correlación entre cada par de factores no contuviera el valor 1 (Anderson \& Gerbing, 1988) y (b) que la varianza promedio extraída para cada factor fuera mayor que el cuadrado de la correlación entre cada par de factores (Fornell \& Larcker, 1981). La Tabla 5 evidencia que todos los casos cumplen con la validez discriminante exceptuando el problema evidenciado entre SP y CFZ, cuya correlación es superior que la varianza promedio extraída.

Aunque la diferencia entre ambos valores es cercana, se procedió a efectuar el test de la diferencia de chi cuadrados entre el modelo de medida y un nuevo modelo en el que se fijó la covarianza entre SP y CFZ a 1 , con el fin de evidenciar si ante un mejor ajuste del modelo la diferencia entre las chi cuadrado era o no significativa (Bagozzi \& Phillips, 1982). La Tabla 6 resume el resultado de dicho test, haciendo las co- rrecciones necesarias al trabajar con el estadístico chi cuadrado de Satorra-Bentler dadas las condiciones de no normalidad de los datos.

El test efectuado evidencia que la diferencia entre las chi cuadrado no es significativa, por lo que se puede afirmar que, a pesar del indicio de complicaciones entre SP y CFZ, el modelo cuenta con validez discriminante.

Posteriormente, se procedió a identificar la fiabilidad del modelo de medida, para lo que se calcularon los alfas de Cronbach para la escala de cada constructo, siendo todos adecuadamente superiores a 0,7 (Nunnally \& Bernstein, 1994). Teniendo presente que el alfa de Cronbach tiende a subestimar la fiabilidad al asumir que todos los ítems están medidos sin error (Bollen, 1989) se procedió a complementar el análisis de fiabilidad con el cálculo del Índice de Fiabilidad Compuesta (IFC) y el Índice de Varianza Extraída (IVE) para cada constructo, que en todos los casos fue superior a 0,5 como lo indica la Tabla 7 .

Tabla 5. Validez discriminante del modelo de medida ${ }^{7}$

\begin{tabular}{|c|c|c|c|c|c|c|}
\hline & FU & UP & A & SP & CFZ & IU \\
\hline FU & 0,820 & 0,661 & 0,553 & 0,482 & 0,503 & 0,607 \\
\hline UP & {$[0,604 ; 0,718]$} & 0,793 & 0,724 & 0,492 & 0,495 & 0,578 \\
\hline A & {$[0,486 ; 0,620]$} & {$[0,675 ; 0,773]$} & 0,899 & 0,468 & 0,517 & 0,536 \\
\hline SP & {$[0,396 ; 0,568]$} & {$[0,408 ; 0,576]$} & {$[0,384 ; 0,552]$} & 0,704 & 0,864 & 0,552 \\
\hline CFZ & {$[0,429 ; 0,577]$} & {$[0,419 ; 0,571]$} & {$[0,444 ; 0,590]$} & {$[0,817 ; 0,911]$} & 0,795 & 0,621 \\
\hline IU & {$[0,544 ; 0,670]$} & {$[0,513 ; 0,643]$} & {$[0,467 ; 0,605]$} & {$[0,474 ; 0,630]$} & {$[0,558 ; 0,684]$} & 0,869 \\
\hline $\begin{array}{l}{ }^{* *} p<, 01 ; ~ \\
\text { diagonal: correlación estimada entre los factores. }\end{array}$ \\
\hline
\end{tabular}

Tabla 6. Test de diferencias de Chi Cuadrado corregido para Chi cuadrados de S-B. ${ }^{8}$

\begin{tabular}{|l|c|}
\hline Chi Cuadrado Modelo Inicial & 1106,800 \\
\hline Grados de Libertad Modelo Inicial & 335 \\
\hline Chi Cuadrado Satorra-Bentler Inicial & 835,570 \\
\hline Chi Cuadrado Modelo covarianza entre SP y CFZ fijada a 1 & 1148,380 \\
\hline Grados de Libertad Modelo Covarianza Fija & 336 \\
\hline Chi Cuadrado Satorra-Bentler Modelo Covarianza Fija & 866,896 \\
\hline Diferencia Chi Cuadrado entre modelos corregida para S-B & 1,358 \\
\hline Probabilidad & $0,244^{*},{ }^{* *}$ \\
\hline${ }^{*}=(p>0,05) ;{ }^{* *}=(p>0,001)$ & \\
\hline
\end{tabular}

\footnotetext{
Fuente: Elaboración propia.
}

Fuente: Elaboración propia. 
Tabla 7. Índices de Fiabilidad ${ }^{9}$

\begin{tabular}{|l|c|c|c|}
\hline \multicolumn{1}{|c|}{ Constructos } & Alfa de Cronbach & $\begin{array}{c}\text { Índice de Fiabilidad } \\
\text { Compuesta (IFC) }\end{array}$ & $\begin{array}{c}\text { Índice de Varianza } \\
\text { Extraída (IVE) }\end{array}$ \\
\hline Facilidad de Uso (FU) & 0,92 & 0,71 & 0,67 \\
\hline Utilidad Percibida (UP) & 0,91 & 0,68 & 0,63 \\
\hline Actitud (A) & 0,94 & 0,82 & 0,81 \\
\hline Seguridad Percibida (SP) & 0,75 & 0,58 & 0,50 \\
\hline Confianza (CFZ) & 0,87 & 0,68 & 0,63 \\
\hline Intención de Uso (IU) & 0,94 & 0,78 & 0,76 \\
\hline
\end{tabular}

Ratificadas la fiabilidad, la validez convergente $y$ discriminante quedan evaluadas las propiedades psicométricas del instrumento de medida satisfactoriamente.

\subsection{Análisis de resultados y contraste de hipótesis}

Se procedió a realizar la estimación del modelo estructural propuesto previamente y que recoge las diversas hipótesis planteadas. Una vez especificado el modelo se continuó con la identificación del mismo, su estimación, evaluación de ajuste, re-especificación e interpretación (Kaplan, 2000). La estimación fue ejecutada mediante el método de máxima verosimilitud robusto, dadas las condiciones de no normalidad de los datos especificada mediante el test de Mardia descrito en la validación de escalas de medida. Nue- vamente se hizo uso del software EQS 6.1. El método de estimación seleccionado sigue las recomendaciones de Joreskog \& Sorbom (1990) para garantizar la consistencia de la estimación del modelo.

La estimación del modelo inicial propuesto arrojó un estadístico chi cuadrado significativo, pero teniendo en cuenta que este estadístico es insuficiente dada su sensibilidad al tamaño muestral (Marsh et al., 1988) se procedió a la verificación de los indicadores de bondad de ajuste, encontrándose valores adecuados superiores a 0,9 en todos los casos y menor a 0,08 en el caso del indicador residual RSMEA. La Tabla 8 recoge la información de los parámetros estimados que conducen al rechazo o no rechazo de las hipótesis propuestas y describe los indicadores de bondad de ajuste observados en el modelo.

Tabla 8. Contraste de hipótesis10

\begin{tabular}{|c|c|c|}
\hline HIPÓTESIS & $\begin{array}{c}\text { Carga } \\
\text { Estandarizada }\end{array}$ & $\begin{array}{c}\text { Valor t } \\
\text { Robusto }\end{array}$ \\
\hline H1: Facilidad percibida de uso $\rightarrow$ Utilidad Percibida & $0,677^{* *}$ & 12,746 \\
\hline H2: Facilidad percibida de uso $\rightarrow$ Actitud hacia la realización de compras/transacciones por internet & n.s. & n.s. \\
\hline H3: Utilidad percibida $\rightarrow$ Actitud hacia la realización de compras/transacciones por internet & $0,599^{* *}$ & 9,740 \\
\hline H4: Utilidad percibida $\rightarrow$ Intención de uso del internet para la realización de compras/transacciones & $0,332^{* *}$ & 5,718 \\
\hline $\begin{array}{l}\text { H5: Actitud hacia la realización de compras/transacciones por internet } \rightarrow \text { Intención de uso de internet para la realización } \\
\text { de compras/transacciones }\end{array}$ & n.s. & n.s. \\
\hline H6: Seguridad Percibida $\rightarrow$ Confianza & $0,887^{\star *}$ & 14,816 \\
\hline H7: Confianza $\rightarrow$ Actitud hacia la realización de compras/transacciones por internet & $0.208^{* *}$ & 4,296 \\
\hline H8: Confianza $\rightarrow$ Intención de uso del internet para la realización de compras/transacciones & $0,432^{\star *}$ & 8,800 \\
\hline
\end{tabular}

Fuente: Elaboración propia.

10 Fuente: Elaboración propia. 
Los resultados obtenidos permiten afirmar que la facilidad de uso influye de forma directa sobre la percepción del individuo acerca de la utilidad del comercio electrónico frente a la compra tradicional $(\mathrm{H} 1$ no rechazada; $\mathrm{B}=0,677 ; \mathrm{p}<0,01)$, mientras que no influye de forma significativa sobre la construcción de la actitud del individuo frente a la realización de compras/transacciones por internet (H2 rechazada). Por su parte la utilidad percibida del uso del comercio electrónico influye tanto en la actitud hacia dicho comportamiento de consumo (H3 no rechazada; $\mathrm{B}=0,599 ; \mathrm{p}<0,01)$ como en la intención del individuo de hacer uso de internet para efectuar compras/ transacciones ( $\mathrm{H} 4$ no rechazada; $\mathrm{B}=0,332 ; \mathrm{p}<0,01$ ).

No se puede afirmar la existencia del rol central de la actitud como paso previo a los comportamientos de los individuos ya que la influencia de dicho factor sobre la intención de uso del comercio electrónico no se evidenció en los resultados (H5 rechazada). Así, las hipótesis del TAM original no son ratificadas en su totalidad, aunque se sostiene la influencia de sus principales antecedentes sobre la intención de uso, de forma directa en el caso de la utilidad percibida y de forma indirecta en el caso de la facilidad percibida de uso (a través de la utilidad percibida).
A su vez, los factores adicionales propuestos para complementar el modelo TAM ratificaron su papel dentro del mismo. La seguridad percibida es un determinante claro de la confianza (H6 no rechazada; $\mathrm{B}=0,887 ; \mathrm{p}<0,01)$, y esta última a su vez influye sobre la actitud (H7 no rechazada; $\mathrm{B}=0,208 ; \mathrm{p}<0,01)$ y directamente sobre la intención de uso del comercio electrónico para la realización de compras/transacciones (H8 no rechazada; $B=0,432 ; p<0,01$ ). La Ilustración 3 ilustra el cumplimiento de las hipótesis propuestas con sus respectivas cargas.

\section{Conclusiones}

La revisión de la literatura permitió identificar que la existencia de estudios académicos acerca del eCommerce es reducida en Colombia y nula en el caso de la aceptación tecnológica. El presente estudio permite llenar vacíos existentes en la literatura académica del contexto colombiano y suramericano en lo relacionado con la aplicación del modelo TAM, ampliamente estudiado en otros contextos, para el estudio del fenómeno de aceptación del comercio electrónico.

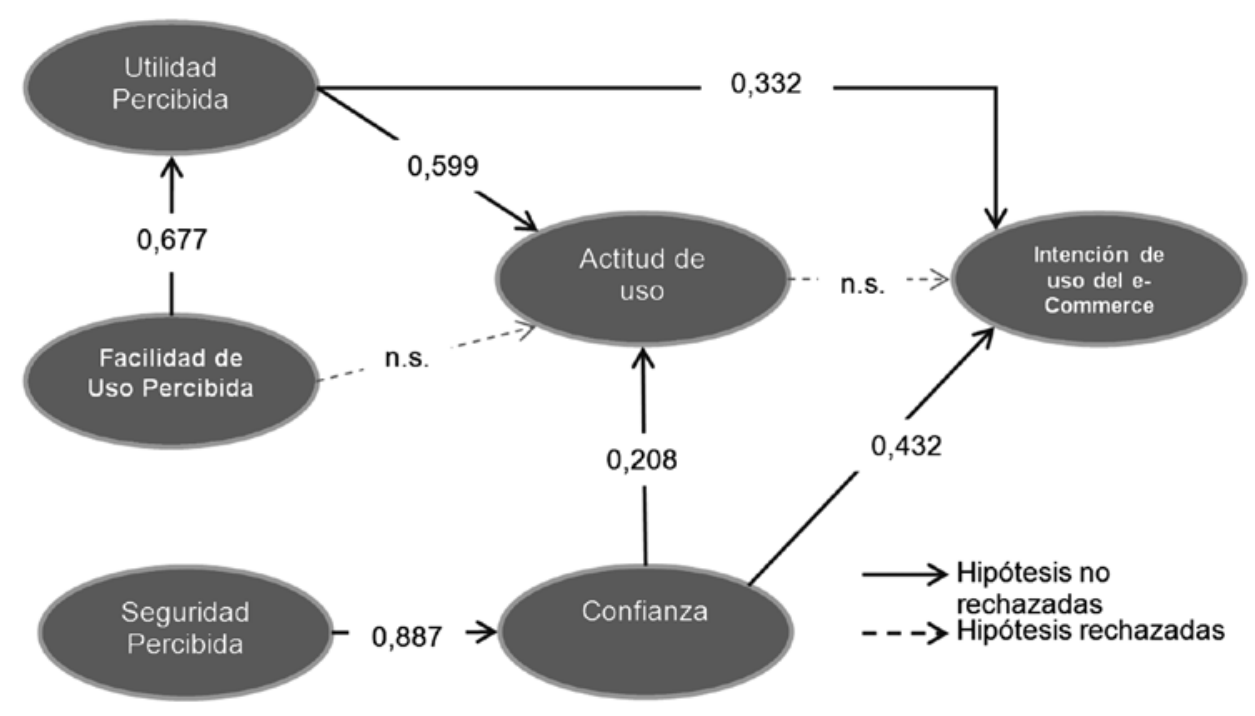

Ilustración 3. Modelo definitivo11 
Dicho modelo tuvo un buen ajuste al ser contrastado con los datos empíricos procedentes de la muestra ratificando la utilidad del TAM como uno de los principales modelos para la investigación de la aceptación tecnológica (Venkatesh \& Davis, 2000).

La facilidad percibida de uso ratificó su importancia como antecedente de la aceptación aunque su influencia se manifestó de forma indirecta a través de la utilidad percibida (Schepers \& Wetzelts, 2006; Venkatesh, 2000). Dichos resultados permiten inferir que la facilidad de uso favorece la utilidad, aunque no influye directamente sobre la actitud, tal como en otros estudios sobre la aceptación de tecnologías digitales (Ruiz et al., 2010).

La utilidad percibida, por su parte, ratificó su influencia directa sobre la actitud y la intención de usar el e-Commerce, sin embargo, la influencia indirecta sobre la intención a través de la actitud no fue ratificada ya que no se encontró una relación significativa entre la actitud y la intención. Lo anterior sugiere la utilización de modelos de aceptación tecnológica basados en el TAM pero que excluyen el constructo de actitud (Venkatesh \& Davis, 2000).

Adicionalmente, se permitió ratificar la línea de influencia de las variables seguridad percibida y confianza como determinantes de la intención de uso del e-Commerce, la primera de forma indirecta a través de la confianza, y la segunda de forma directa. La confianza se identificó como el principal antecedente de la intención de uso del e-Commerce, ratificando los hallazgos del estudio de Palvia (2009). Otros estudios (Suh \& Han, 2003) también ratifican la influencia significativa de la confianza sobre la intención.

En cuanto a las implicaciones gerenciales, el estudio permite identificar que la generación de confianza es de vital importancia para el éxito de las estrategias de comercialización de productos y servicios a través de las plataformas en línea. La utilización de referentes de seguridad para el pago, como lo pueden ser PSE y Todo1 en el caso colombiano o PayPal \& Google Checkout a nivel internacional, favorecen la percepción de seguridad del cliente, al igual que la comercialización de productos de marcas reconocidas, dado que se reducen los riesgos asociados al producto.

La facilidad de uso y la utilidad percibida deben ser criterios de alta importancia a la hora de diseñar la interacción de las tiendas en línea con los clientes. Se deben favorecer las rutas cortas para que los clientes puedan acceder a la información o productos/ servicios buscados. También debe asegurarse que existan atributos de diferenciación sobre el comercio tradicional, como lo pueden ser el tiempo, un menor precio, la asesoría en línea o el acceso a información detallada del producto.

Para futuras investigaciones se sugiere la utilización de nuevas variables que pueden usarse para proponer nuevos modelos de aceptación del e-Commerce, como las procedentes de la Teoría del Comportamiento Planificado o de la Teoría de Usos y Gratificaciones de los medios de comunicación. Adicionalmente, la inclusión de variables relacionadas con los individuos, tales como la experiencia de uso o la capacidad de innovación (innovativeness).

Por último, se sugiere también la aplicación de modelos similares a otras tecnologías de comercio electrónico específicas como el e-Banking o el comercio a través de dispositivos móviles (m-Commerce).

\section{Referencias}

Ajzen, I. (1991) "The Theory of Planned Behavior". En: Organizational Behavior and Human Decision Processes, 50 (2): 179-211.

Ajzen, I. \& Fishbein, M. (1977) "Attitude-Behaviour relations: A theoretical analysis and review of empirical research". En: Psychological Bulletin, 84 (5): 888-918.

Ajzen, I. \& Gilbert, N. (2008) "Attitudes and the prediction of behavior" En: Crano, W. D. y Prislin R. (Eds.). Attitudes and attitude change. New York: Psychology Press.

Al-Gahtani, S. (2011) "Modeling the electronic transactions acceptance using an extended technology acceptance model". En: Applied Computing and Informatics, 9 (1): 47-77.

Anderson, J. \& Gerbing, D (1988) "Structural equation modeling in practice: A review and recommended two-step approach". En: Psychological Bulletin, (103): 411-423.

Bagozzi, R. P. (1992) "The Self-Regulation of attitudes, intentions, and behavior". En: Social Psychology Quarterly, 55 (2): 178-204.

Bagozzi, R. P. \& Phillips L. W. (1982) "Representing and Testing Organizational Theories: A Holistic Construal". En: Administrative Science Quarterly, 27 (3): 459-489. 
Bagozzi, R. P. \& Yi, Y. (1988) "On the evaluation of structural equation models". En: Journal of Academy of Marketing Science, 16 (1):74-94.

Bigné, E.; Ruiz C. \& Sanz S. (2007) "Key Drivers of Mobile Commerce Adoption. An Exploratory Study of Spanish Mobile Users". En: Journal of Theoretical and Applied Electronic Commerce Research, 2 (2): 48.

Bollen, K. A. (1989) Structural equations with latent variables. Mexico: Wiley.

Brynjolfsson, E. \& Smith M. D. (2000) "Frictionless Commerce? A Comparison of Internet and Conventional Retailers". En: Management Science, 46 (4): 563-585.

Castells, M. (2000) "Internet y la sociedad red". Disponible en: http://www.uoc.edu/web/cat/articles/castells/print.html].

Coyle, J. \& Thorson, R.. (2001) "The effects of progressive levels of interactivity and vividness in Web marketing sites". En: Journal of Advertising, 30 (3): 65.

Chang, H. \& Chen S. (2009) "Consumer perception of interface quality, security, and loyalty in electronic commerce". En: Information \& Management, 46 (7): 411.

Davis, F. D. (1989) "Perceived usefulness, perceived ease of use, and user acceptance of information technologies". En: MIS Quarterly, 13 (3): 21.

Davis, F. D.; Bagozzi, R. \& Warshaw, P. (1989) "User Acceptance of Computer Technology: A comparisson of two theoretical models". En: Management Science, 35 (8): 982.

Deutsch, M. (1958) "Trust and suspicion". En: Journal of Conflict Resolution, 2 (4): 265-279.

Doney, P., Barry J. \& Abratt, R. (2007) "Trust determinants and outcomes in global B2B services". En: European Journal of Marketing, 41 (9/10): 1096-1116.

Fishbein, M. \& Ajzen, I. (1975) Belief, attitude, intention, and behavior: An introduction to theory and research. Reding Mass: Addison-Wesley.

Fornell, C. \& Larcker, D. (1981) "Evaluating structural equation models with unobservable variables and measurement error". En: Journal of Marketing Research, 18 (1): 39-50.

Garrity, E.; O'Donnell, J.; Kim, Y. \& Sanders, G. (2007) "An Extrinsic and Intrinsic Motivation-Based Model for Measuring Consumer Shopping Oriented Web Site Success". En: Journal of Electronic Commerce in Organizations, 5 (4): 18.

Gefen, D. (2000) "E-commerce: the role of familiarity and trust". En: Omega, 28 (6): 725-737.

Gefen, D.; Karahanna, E. \& Straub, D. (2003) "Inexperience and experience with online stores: The importance of TAM and trust". En: IEEE Transactions on Engineering Management, 50 (3): 307-322.

Gefen, D. \& Straub, D. (2004) "Consumer trust in B2C e-Commerce and the importance of social presence: experiments in eProducts and e-Services". En: Omega, 32 (3): 407-424.

Grewal, D. \& Levy, M. (2009) "Emerging Issues in Retailing Research". Journal of Retailing, 85 (4): 522-522-526.

Hair, J.; Anderson, R.; Tatham, R. \& Black, W. (2001). Análisis multivariante (5a ed.). Madrid: Prentice Hall Iberia.

Ji-Won, M. \& Young-Gul, K. (2001) "Extending the TAM for a WorldWide-Web context". En: Information \& Management, 38 (4): 217.

Jones, K. \& Leonard, L. (2007) "Consumer-to-Consumer Electronic Commerce: A Distinct Research Stream". En: Journal of Electronic Commerce in Organizations, 5 (4): 39.

Joreskog, K. \& Sorbom, D. (1990) LISREL 7. A guide to program and application. Michigan: SPSS.

Kaplan, D. (2000) Structural Equation Modeling. Foundations and Extensions. . Thousand Oaks: SAGE Publications.

Khalifa, M. \& Shen, K. N. (2008) "Explaining the adoption of transactional B2C mobile commerce". En: Journal of Enterprise Information Management, 21 (2): 110.

Kim, D. ; Ferrin, D. L. \& Rao, H. (2008) "A trust-based consumer decision-making model in electronic commerce: The role of trust, perceived risk, and their antecedents". En: Decision Support Systems, 44 (2): 544-564.

Kim, M.; Chung N. \& Lee C, K. (2011) "The effect of perceived trust on electronic commerce: Shopping online for tourism products and services in South Korea". En: Tourism Management, 32 (2): 256-265

Kim, T. \& Biocca F. (1997) "Telepresence via Television: Two Dimensions of Telepresence May Have Different Connections to Memory and Persuasion," Disponible en: http://jcmc.indiana. edu/vol3/issue2/kim.html\#Footnote1].

King, W. \& He, J. (2006) "A meta-analysis of the technology acceptance model". En: Information \& Management, (43): 16.

Kleijnen, M.; Wetzels, M. \& de Ruyter, K. (2004) "Consumer acceptance of wireless finance". En: Journal of Financial Services Marketing, 8 (3): 206.

Kotler, P. \& Armstrong, G. (2003) Fundamentos de Marketing (6a ed.). México: Pearson.

Kshetri, N. (2007) "Barriers to e-commerce and competitive business models in developing countries: A case study". En: Electronic Commerce Research and Applications, 6 (4): 443.

Lassar, W.; Manolis, C. \& Lassar, S. (2005) "The relationship between consumer innovativeness, personal characteristics, and online banking adoption". En: The International Journal of Bank Marketing, 23 (2/3): 176.

Liu, C.; Marchewka, J.; Lu J. \& Yu, C.S. (2005) "Beyond concern - a privacy-trust-behavioral intention model of electronic commerce". En: Information \& Management, 42 (2): 289-304.

Lu', J.; Yu C.S.; Liu C. \& Yao, J. (2003) "Technology acceptance model for wireless Internet". En: Internet Research, 13 (3): 206

Luarn, P. \& Lin, H. (2005) "Toward an understanding of the behavioral intention to use mobile banking". En: Computers in Human Behavior, 21 (6): 873-891.

Marsh, H.; Balla J. \& McDonald, R. (1988) "Goodness-of-fit indices in confirmatory factor analysis: The effect of sample size". En: Psychological Bulletin, 102 (3): 391-410.

Mathieson, K.; Peacock, E. \& Chin W. (2001) "Extending the technology acceptance model: The influence of perceived user resources". En: Database for Advances in Information Systems, 32 (3): 86

Mejía, J. (2011) Penetración de usuarios de Internet en Colombia: segundo país de Latinoamérica. Nota Web disponible en http:// www.ecbloguer.com/marketingdigital/?p=640].

Ministerio de Tecnologías de la Información y las Comunicaciones, $R$. d. C. (2011) Boletín trimestral de las TIC - Conectividad - Cifras primer trimestre de 2011. Bogotá: Ministerio de Tecnologías de la Información y las Comunicaciones.

Moore, G. \& Benbasat, I. (1991) "Development of an instrument to measure the perception of adopting an information technology innovation". En: Information Systems Research, 2 (3): 199-222.

Moorman, C.; Deshpande, R. \& Zaltman G. (1993) "Factors affecting trust in market research relationships". En: Journal of Marketing, 57 (1): 81. 
Moorman, C.; Zaltman, G. \& Desphandé, R. (1992) "Relationships between providers and users of market research: The dynamics of trust within and between organizations". En: Journal of Marketing Research, 29 (3): 314-329.

Netsize (2008) The Netsize guide 2008. Información corporative de NETSIZE -Gemalto Corporation disponible en http://www. netsize.com

Nunnally, J. \& Bernstein, I. (1994) Psychometric Theory (3a ed.). New York: McGraw-Hill.

Palvia, P. (2009) "The role of trust in e-commerce relational exchange: A unified model ". En: Information \& Management, 46 (4): 213-220.

Pavlou, P. (2001)"Consumer Intentions to Adopt Electronic Commerce - Incorporating Trust and Risk in the Technology Acceptance Model". En: DIGIT 2001 Proceedings. Paper 2.

Robinson, J.; Marshall, G. \& Stamps, M. (2005) "Sales force use of technology: antecedents to technology acceptance". En: Journal of Business Research, 58 (12): 1623-1631.

Rodríguez del Bosque, I. \& Herrero, A. (2007) "Antecedentes de la utilidad percibida en la adopción del comercio electrónico entre particulares y empresas". Cuadernos de Economía y Dirección de la Empresa, 34 (2): 107-134.

Ruiz, M.; Sanz, B. \& Tavera, J. (2010) "A comparative study of mobile messaging services acceptance to participate in television programmes". En: Journal of Service Management, 21 (1): 69-102.

Savitskie, K.; Royne, M.; Persinger, S.; Grunhagen, M. \& Witte, C. (2007) "Norwegian Internet Shopping Sites: An Application \& Extension of the Technology Acceptance Model". En: Journal of Global Information Technology Management, 10 (4): 54.

Schepers, J. \& Wetzels, M. (2006) "A meta-analysis of the technology acceptance model:

Investigating subjective norm and moderation effects". En: Information \& Management, 44 (3): 90-103

Shih, H. (2004) "An empirical study on predicting user acceptance of e-shopping on the Web". En: Information \& Management, 41 (3): 351.

Simon, S.; John, S.; Roula, M. \& John, H. (2006) "Technology acceptance and $\mathrm{m}$-commerce in an operational environment". En. Journal of Enterprise Information Management, 19 (5): 525.

Singh, N.; Fassott, G.; Chao, M. \& Hoffmann, J. (2006) "Understanding international web site usage: A cross-national study of German, Brazilian, and Taiwanese online consumers". En: International Marketing Review, 23 (1): 83.
Suh, B. \& Han, I. (2003) "The impact of trust and perception of security control on the acceptance of electronic commerce". En: International Journal of Electronic Commerce, 7 (3): 135-161.

Taylor, S. \& Todd P. (1995) "Understanding information technology usage: A test of competing models". En: Information Systems Research, 6 (2): 144.

Uriel, E. \& Aldás J. (2005) Análisis multivariante aplicado: Aplicaciones al marketing, investigación de mercados, economía, dirección de empresas y turismo (1a ed.). Madrid: Thomson Paraninfo.

Varadarajan, P. \& Yadav, M. (2002) "Marketing Strategy and the Internet: An Organizing Framework". En: Academy of Marketing Science Journal, 30 (4): 296.

Venkatesh, V. (2000): "Determinants of perceived ease of use: Integrating control, intrinsic motivation, and emotion into the technology acceptance model". Information Systems Research, 11 (4):342.

Venkatesh, V. \& Davis, F. (1996) "A model of the antecedents of perceived ease of use: Development and test". En: Decision Sciences, 27 (3):451.

Venkatesh, V. \& Davis, F. (2000) "A theoretical extension of the technology acceptance model: Four longitudinal field studies". En: Management Science, 46 (2):186.

Venkatesh, V.; Morris, M.; Davis, G. \& Davis, F. (2003) "User Acceptance of Information Technology: Toward a unified view". En: MIS Quarterly, 27 (3): 425-478.

Wang, Y.; Lin, H. \& Luarn, P. (2006) "Predicting consumer intention to use mobile service". En: Information Systems Journal, 16 (2): 157.

Wu, J. \& Wang S. (2005) "What drives mobile commerce? An empirical evaluation of the revised technology acceptance model". En: Information \& Management, 42 (5): 719.

Xiaoni, Z.; Victor, R. \& Chang, E. (2006) "The Role of Impulsiveness in a TAM-Based Online Purchasing Behavior Model". En: Information Resources Management Journal, 19 (2): 54.

Yang, K. (2005) "Exploring factors affecting the adoption of mobile commerce in Singapore". En: Telematics and Informatics, 22 (3): 257-277.

Yang, K. (2007) "Exploring Factors Affecting Consumer Intention to Use Mobile Advertising in Taiwan". En: Journal of International Consumer Marketing, 20 (1): 33-49.

Yu, J.; Ha, I.; Choi, M. \& Rho, J. (2005) "Extending the TAM for a t-commerce". En: Information \& Management, 42 (7): 965-976. 
